\title{
Prognostic Role of Circular RNAs Expression in Bladder Carcinoma: A Meta-Analysis
}

\author{
Siyuan Wang, Shengqiang Fu, Qiang Chen, Yutang Liu, Zhilong Li, and Ting Sun
}

Background: Circular RNAs (circRNAs), a type of noncoding RNA, are associated with the occurrence and development of cancers. In recent years, their potential as biomarkers for bladder cancer (BC) has attracted increased attention.

Aims: To reveal the value of circRNAs in the prognosis of BC.

Methods: Relevant studies were collected to extract the clinicopathological data and overall survival (OS) of patients with BC. We then evaluated their tumor-related clinicopathological indicators using pooled odds ratio (OR) and 95\% confidence interval (CI). Hazard ratio (HR) and 95\% CI were used to estimate the OS of BC patients who expressed abnormal circRNAs.

Results: The results indicate that seven circRNAs (circMYLK, circASXL1, hsa_circ_0000144, circ-VANGL1, circGprc5a, circZFR, and circ-cTFRC) whose expression was upregulated in tumor tissues, are associated with poor clinicopathological features (tumor/node/metastasis [TNM] stage: OR=4.86, 95\% CI: 2.53-9.34; histological grade: $\mathrm{OR}=3.71,95 \% \mathrm{CI}$ : 2.37-5.79; lymph node metastasis: $\mathrm{OR}=3.23$; 95\% CI: 2.15-4.88; recurrence: $\mathrm{OR}=5.33 ; 95 \% \mathrm{CI}: 2.27-12.52$ ) and poor prognoses (OS: $\mathrm{HR}=1.69 ; 95 \% \mathrm{CI}: 1.17-2.44)$. We also found that seven circRNAs (circMTO1, circ-ITCH, circUBXN7, circFNDC3B, circ-ZKSCAN1, circPICALM, and circACVR2A) that were downregulated in tumors were associated with better clinicopathological indicators (TNM stage: $\mathrm{OR}=0.23,95 \% \mathrm{CI}: 0.14-0.38$; histological grade: $\mathrm{OR}=0.21,95 \% \mathrm{CI}: 0.13-0.33$; lymph node metastasis: $\mathrm{OR}=0.26$; 95\% CI: $0.15-0.47$ ) and better prognoses (OS: HR $=0.42 ; 95 \%$ CI: $0.30-0.58$ ).

Conclusion: Our results suggest that certain circRNAs are related to the clinicopathological characteristics and prognosis of $\mathrm{BC}$ patients, and may potentially be used as biomarkers.

Keywords: circular RNA, bladder cancer, prognosis, meta-analysis

\section{Introduction}

B LADDER CANCER (BC) was ranked the ninth-most prevalent cancer in the world (Antoni et al., 2017). In 2012, a total of 429,793 patients were diagnosed with BC, and 165,084 deaths occurred globally (Siegel et al., 2017). BC cases are classified according to the depth of tumor infiltration (Burger et al., 2013). Newly diagnosed BC cases comprised $75 \%$ nonmuscle invasive bladder cancer (MIBC) and $25 \%$ MIBC. MIBC patients typically present with local or distant metastases and poor prognoses. Therefore, it is urgent to find useful molecular biomarkers for the diagnosis and prognosis of newly identified BC cases.

Noncoding RNAs (ncRNAs), include several classes of transcripts, are encoded by the genome, but are not translated into proteins. Recently, with the development of next- generation RNA sequencing technologies, many cancerrelated ncRNAs have been discovered (Slack and Chinnaiyan, 2019). Among them, microRNAs (miRNAs), long noncoding RNAs (lncRNAs), and circular RNAs (circRNAs) have been associated with clinical outcomes. miRNAs are short ncRNAs ( $\sim 22 \mathrm{nt})$ that regulate the expression of other RNAs via binding with complementary sequences in target mRNAs (Gebert and MacRae, 2019). IncRNAs, sequence length $>200$ bp, have been shown to play important roles in cancer through chromatin modification and transcriptional and posttranscriptional regulation of mRNAs (Yao et al., 2019). circRNAs are primarily produced by the splicing process for the production of mature mRNAs and are characterized by a covalent closed-loop structure that has neither a $5^{\prime}$ cap end nor a 3' polyadenylation tail (Salzman, 2016). Studies have confirmed that some abnormally expressed circRNAs can affect

Department of Urology, The First Affiliated Hospital of Nanchang University, Nanchang, Jiangxi, China. 
the development of tumors by regulating the expression of target genes and might be suitable as cancer biomarkers (Meng et al., 2017; Wang et al., 2018b). Salmena et al. (2011) proposed the competitive endogenous RNA (ceRNAs) hypothesis linking the expression of mRNAs, miRNAs, IncRNAs, and circRNAs together into a complex network. This theory proposes that lncRNAs or circRNAs can serve as "molecular sponges" of miRNAs to decrease the latter's activity, thereby upregulating miRNA target gene expression. In support of this theory, an ever-increasing number of circRNAs have been shown to regulate gene expression via such ceRNA networks in numerous tumors (Wang et al., 2018a; Cong et al., 2019). For example, circ-tau tubulin kinase 2 (TTBK2) competitively binds miRNA-217 and upregulates the expression of the oncogene HNF1 homeobox B (HNF1 $\beta$ ) to promote proliferation, invasion, and migration of glioma cells (Zheng et al., 2017). In lung adenocarcinoma, circ-mitochondrial TRNA translation optimization 1 (circMTO1) serves as a sponge of oncogenic miRNA-17 to increase the expression of the RNA-binding protein QKI-5, leading to the inactivation of the Notch signaling pathway, thereby restraining the growth of lung adenocarcinomas (Zhang et al., 2019a). In BC, circ-myosin light chain kinase (circMYLK) binds to miRNA-29a to activate the VEGFA/VEGFR and downstream Ras/ERK signaling pathways to promote the proliferation, migration, and vascularization of BC cells (Zhong et al., 2017). Although there are multiple studies that have been undertaken at the cell and organismal level (Cong et al., 2019; Li et al., 2019b), it is unclear whether the abnormally expressed circRNAs are related to the clinicopathology of BC patients and whether they have a clear diagnostic and prognostic value. Due to the fact that the data provided by individual studies of circRNAs in BC were insufficient to analyze their diagnostic and prognostic roles, we conducted a meta-analysis.

\section{Materials and Methods}

\section{Study selection}

We searched for the following search terms: ("circRNA" OR "circular RNA") AND ("bladder cancer" OR "bladder tumor" OR "bladder carcinoma" OR "bladder neoplasm") in PubMed, EMBASE, Web of Science, and Cochrane Library before November 21, 2019. We selected studies using the following inclusion criteria: (1) literature findings that included data on abnormally expressed circRNAs and survival outcomes in BC patients; (2) the study that indicated that the included patients were pathologically diagnosed as having $\mathrm{BC}$; and (3) the studies that investigated the clinicopathological features of circRNAs in BC. Exclusion criteria were as follows: (1) results of the literature studies were not published in English; (2) similar studies from the same author reusing clinical specimens; and (3) reviews, meta-analyses, and case reports.

\section{Data extraction}

First, S.W. and S.F. rigorously evaluated the eligibility of the included studies, and then extracted data from each study including the following: (1) the first author, the year of publication, the circRNA that was abnormally expressed, the related miRNA(s), detection methods, and the presumptive regulatory role of the circRNA; (2) clinicopathological information regarding circRNA expression, age, gender, tumor size, tumor/node/metastasis (TNM) stage, histological grade, lymphatic metastasis, vascular invasion, and recurrence; (3) prognostic data including follow-up time, hazard ratio (HR), and $95 \%$ confidence interval (CI) of BC patients.

\section{Quality assessment}

The Newcastle-Ottawa Scale (NOS) was used to determine the quality of the literature included in this meta-analysis (Stang, 2010).

\section{Statistical analyses}

We used pooled odds ratios (OR) and 95\% CI to evaluate $\mathrm{BC}$ clinicopathological indicators; we used the HR and 95\% CIs to evaluate overall survival (OS) of BC. Heterogeneity among the included studies was tested using $I^{2}$ tests. When $I^{2}$ $<50 \%$, there was considered to be no significant heterogeneity, a fixed effect model was used for this analysis.

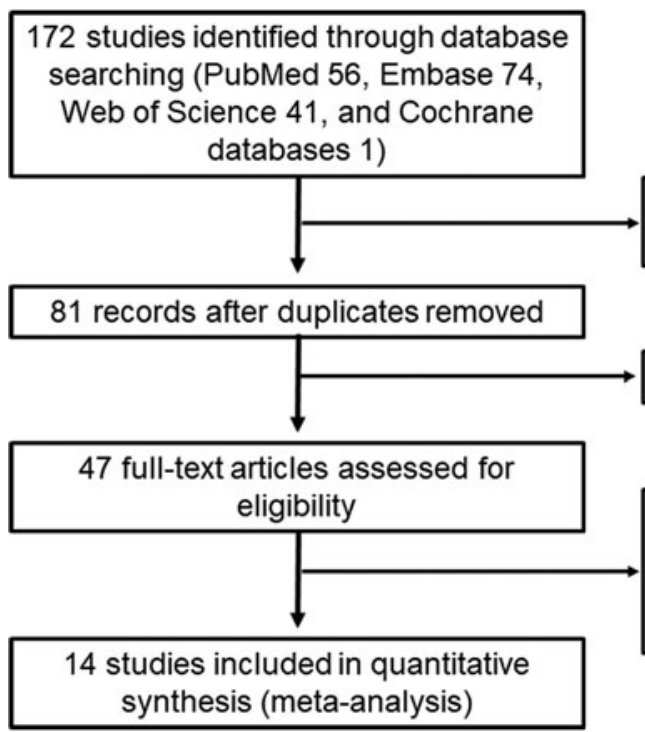

91 duplicated studies excluded

34 records excluded

33 full-text articles excluded (No outcomes 23, Review 4, Animal articles 3, Insufficient data 3)
FIG. 1. Flowchart of the research selection process. 
Otherwise, a random-effect model was used (Egger et al., 1997). Sensitivity analysis was used to check the consistency of the overall effect assessment, and Begg's test was used to analyze the publication bias of the study. A $p$-value $<0.05$ was considered statistically significant.

\section{Results}

\section{Search results}

As shown in Figure 1, the process of selecting the included literature was as follows: 172 records of circRNA expression and BC were found: PubMed (56), EMBASE (74), Web of Science (41), and Cochrane databases (1). Among them, 91 duplicate publications were deleted. After filtering through titles and abstracts, 34 records were excluded. The remaining 47 articles were evaluated for eligibility via reading of the full text; 33 were excluded due to insufficient data. A total of 14 studies were included in the final meta-analysis.

\section{Study characteristics and study quality}

The baseline data from the 14 included studies are shown in Table 1. These studies covered a total of 14 circRNAs spread among 1430 patients who were pathologically diagnosed as suffering from BC in Asia. Seven of these circRNAs were associated with poor clinicopathological outcomes (Tang et al., 2017; Zhong et al., 2017; Gu et al., 2018; Huang et al., 2018; Su et al., 2019; Zeng et al., 2019; Zhang et al., 2019c); and the remaining seven were associated with better clinicopathological outcomes (Liu et al., 2018a, 2018b; Yang et al., 2018; Bi et al., 2019; Dong et al., 2019; Li et al., 2019a; Yan et al., 2019). In addition, our baseline data also included the miRNA levels reported in the primary studies that are thought to be part of a ceRNA network that regulates the expression of tumor genes. Follow-up times ranged from 44 to 84 months. The NOS was used to evaluate the quality of the included studies. All 14 of the included studies received scores that were $>7$ points, indicating that the quality of the studies included in this meta-analysis is high, and likely ensuring the reliability of the overall results (Fig. 2).

\section{Meta-analysis for clinicopathological features}

The relationships between the circRNA levels and the BC clinicopathological characteristics are shown in Table 2. We observed that higher levels of seven circRNAs (circMYLK, circASXL1, hsa_circ_0000144, Circ-VANGL1, circGprc5a, circZFR, and circ-cTFRC) were associated with poor clinicopathological outcomes (TNM stage: $\mathrm{OR}=4.86,95 \% \mathrm{CI}$ : 2.53-9.34; histological grade: $\mathrm{OR}=3.71,95 \% \mathrm{CI}: 2.37-5.79$; lymph node metastasis: $\mathrm{OR}=3.23$; $95 \% \mathrm{CI}$ : $2.15-4.88$; recurrence: $\mathrm{OR}=5.33 ; 95 \% \mathrm{CI}: 2.27-12.52$, all $p<0.05$ ). Furthermore, the results also showed that seven circRNAs (circMTO1, circ-ITCH, circUBXN7, circFNDC3B, circZKSCAN1, circPICALM, and circACVR2A) with lower expression levels were associated with better clinicopathological results (TNM stage: $\mathrm{OR}=0.23,95 \% \mathrm{CI}$ : $0.14-0.38$; histological grade: $\mathrm{OR}=0.21,95 \% \mathrm{CI}: 0.13-0.33$; lymph node metastasis: $\mathrm{OR}=0.26$; 95\% CI: 0.15-0.47, all $p<0.05$ ). In contrast, we did not observe significant correlations for age, gender, tumor size, and vascular invasion $(p>0.05)$.

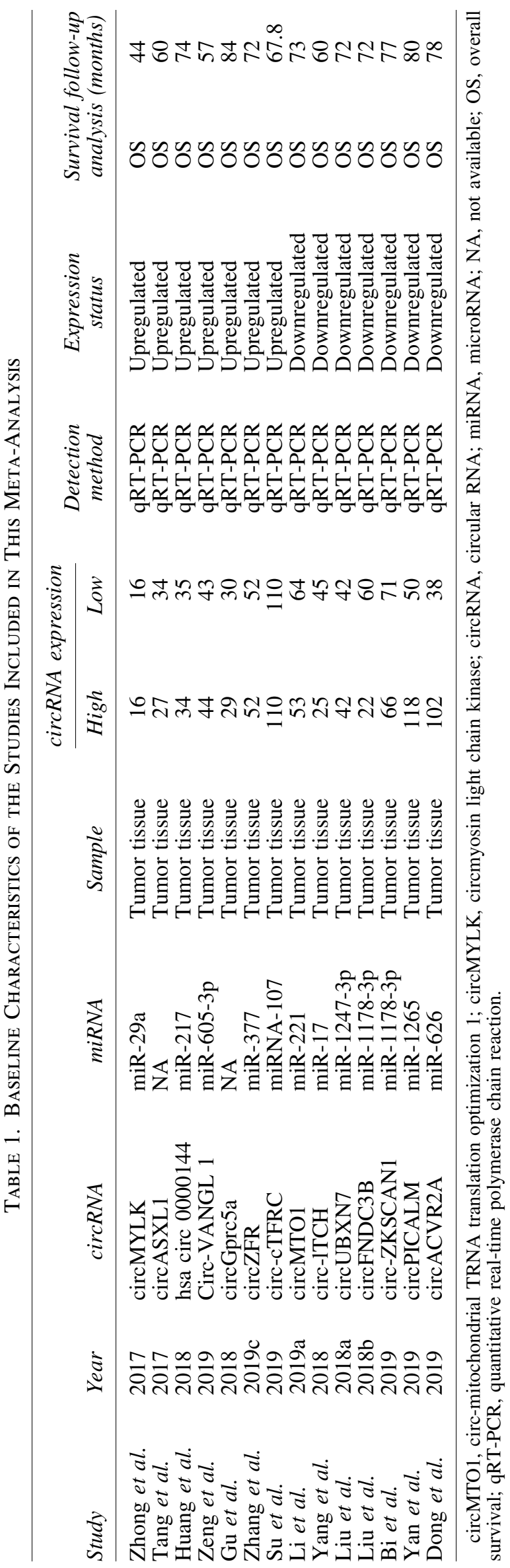




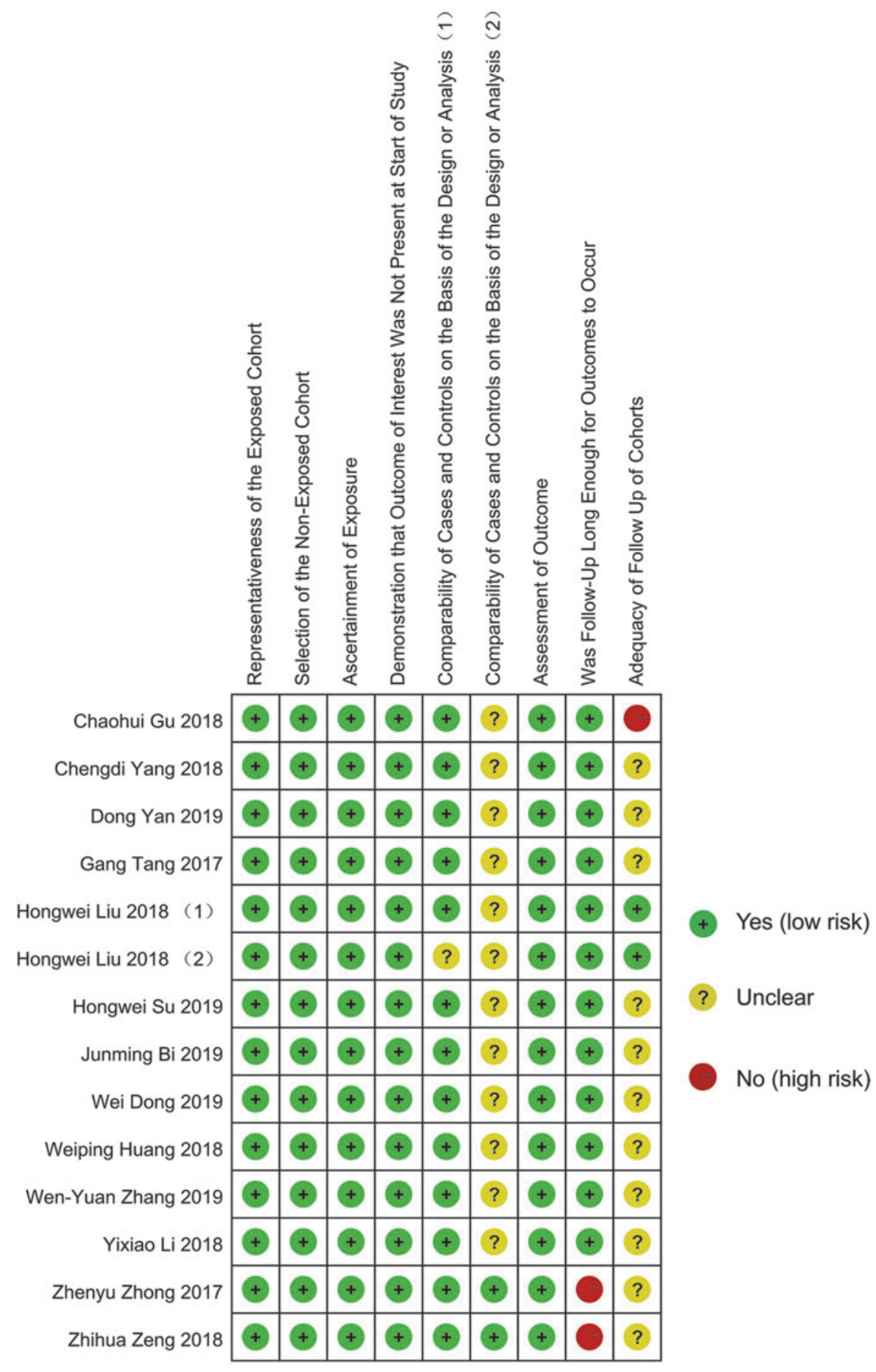

FIG. 2. The Newcastle-Ottawa Scale assesses the quality of studies included.

\section{Meta-analysis for OS}

Our results show that the circRNAs that are abnormally expressed in BC patients have significant prognostic value. One such value is that seven of the circRNA (circMYLK, circASXL1, hsa_circ_0000144, Circ-VANGL1, circGprc5a, circZFR, and circ-cTFRC) expression levels were inversely related to $\mathrm{OS}$ of $\mathrm{BC}$ patients $(\mathrm{HR}=1.69 ; 95 \% \mathrm{CI}$ : $1.17-2.44$; $p=0.005$ ) (Fig. 3A). Another is that seven different circRNA (circMTO1, circ-ITCH, circUBXN7, circFNDC3B, circZKSCAN1, circPICALM, and circACVR2A) expression levels were positively correlated with the OS of BC patients $(\mathrm{HR}=0.42$; 95\% CI: $0.30-0.58 ; p<0.001)$ (Fig. 3B). The statistical results show that the various circRNAs had no significant heterogeneity $\left(I^{2}=0.0 \%, p=0.945 ; I^{2}=45.1 \%\right.$, $p=0.091$ ), and thus, we used a fixed-effect model for the analysis.

\section{Sensitivity analysis and publication bias}

As shown in Figure 4 when the included studies were deleted one by one, our sensitivity analysis results showed that 
Table 2. Clinical Characteristics of Circular RNAs in Bladder Cancer

\begin{tabular}{|c|c|c|c|c|c|c|}
\hline & \multicolumn{2}{|c|}{ Tumor promoter } & \multirow[b]{2}{*}{$\mathrm{p}$} & \multicolumn{2}{|c|}{ Tumor suppressor } & \multirow[b]{2}{*}{$\mathrm{p}$} \\
\hline & $O R$ & $95 \% \mathrm{CI}$ & & $O R$ & $95 \% C I$ & \\
\hline Age & 1.49 & $0.88-2.50$ & 0.14 & 0.83 & $0.57-1.20$ & 0.32 \\
\hline Gender (male/female) & 1.25 & $0.80-1.95$ & 0.32 & 1.37 & $0.94-2.00$ & 0.10 \\
\hline Tumor size $(\geq 3 /<3)$ & 0.59 & $0.33-1.06$ & 0.08 & 0.99 & $0.59-1.68$ & 0.98 \\
\hline TNM stage pT2-pT4/pTa-pT1 & 4.86 & $2.53-9.34$ & 0.000 & 0.23 & $0.14-0.38$ & 0.000 \\
\hline Histological grade & 3.71 & $2.37-5.79$ & 0.000 & 0.21 & $0.13-0.33$ & 0.000 \\
\hline Lymph node metastasis $(\mathrm{Y} / \mathrm{N})$ & 3.23 & $2.15-4.88$ & 0.000 & 0.26 & $0.15-0.47$ & 0.000 \\
\hline Vascular invasion $(\mathrm{Y} / \mathrm{N})$ & 0.88 & $0.32-2.38$ & 0.80 & 0.58 & $0.19-1.77$ & 0.33 \\
\hline Recurrence $(\mathrm{Y} / \mathrm{N})$ & 5.33 & $2.27-12.52$ & 0.000 & 1.23 & $0.40-3.75$ & 0.71 \\
\hline
\end{tabular}

CI, confidence interval; N, no; OR, odds ratio; TNM, tumor/node/metastasis; Y, yes.

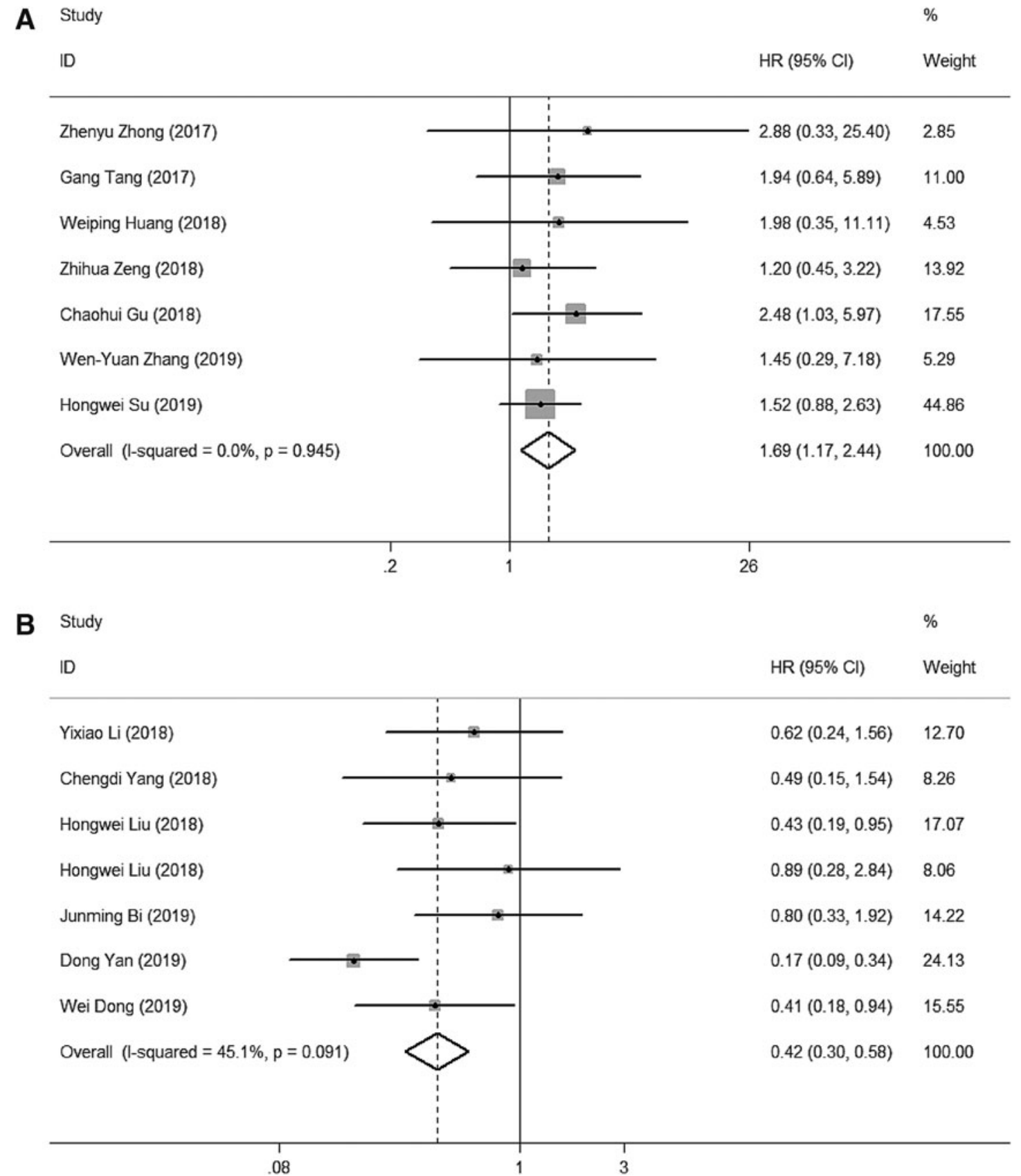

FIG. 3. Forest plot of circRNA expression and OS in BC patients. (A) Forest plot of tumor-promoting circRNAs. (B) Forest plot of tumor-inhibiting circRNAs. BC, bladder carcinoma; CI, confidence interval; circRNA, circular RNA; HR, hazard ratio, OS, overall survival. 

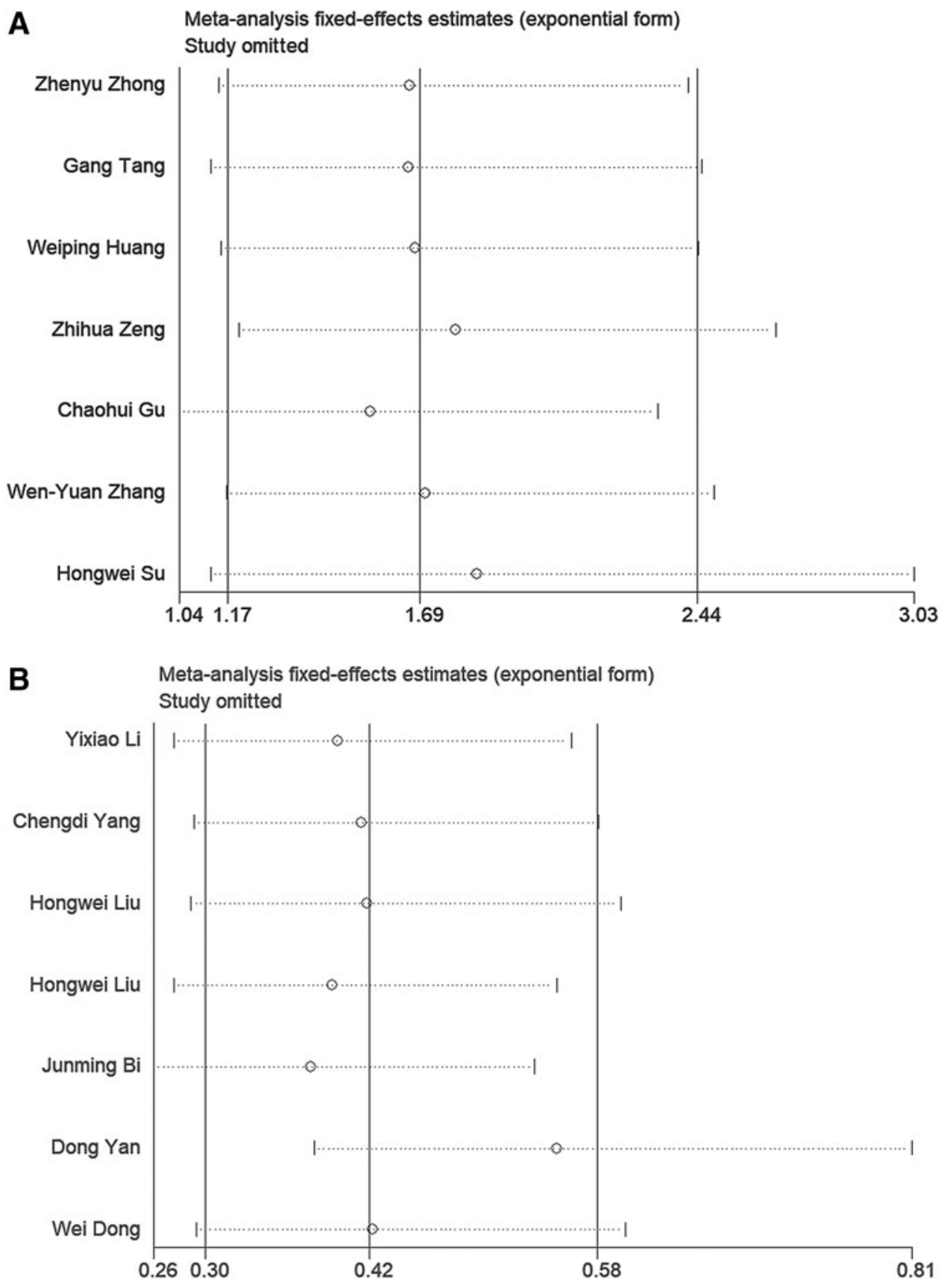

FIG. 4. Sensitivity analysis of circRNAs in predicting OS outliers in BC patients. (A) Tumor-promoting circRNAs; (B) tumor-inhibiting circRNAs. the pooled HR results from the remaining studies for OS did not change. Results based on Begg's funnel plots show that there was no significant publication bias (Fig. 5A, B, $p>0.05)$.

\section{Discussion}

BC is characterized by both high morbidity and mortality (Martinez Rodriguez et al., 2017). It remains a leading cause of cancer-related deaths because of distant metastases and high recurrence rates (Di Meo et al., 2017). Surgery and postoperative chemotherapy are the primary treatments. Although the treatment of $\mathrm{BC}$ has improved somewhat due to surgical techniques and second-generation chemotherapy drugs, the overall treatment effect has not significantly improved due to its biological characteristics (Martinez Rodriguez et al., 2017). This highlights the urgent need for novel biomarkers to more accurately predict its recurrence and to aid in the choice of treatment regimens.

circRNAs were discovered in 1976 and were originally thought to be by-products of splicing precursor mRNAs (Sanger et al., 1976). With the development of highthroughput and bioinformatic technologies, circRNAs have been proven to be widely expressed in various organisms, and to have tissue and cell specificity (Patop et al., 2019). Highthroughput sequencing of oral squamous cell carcinoma RNAs has shown that circRNAs are widely expressed in both cancerous tissues and the normal oral mucosa tissues. Chen 
FIG. 5. The Begg's test evaluated the publication bias of this metaanalysis. (A) Tumor-promoting circRNAs; (B) tumor-inhibiting circRNAs.

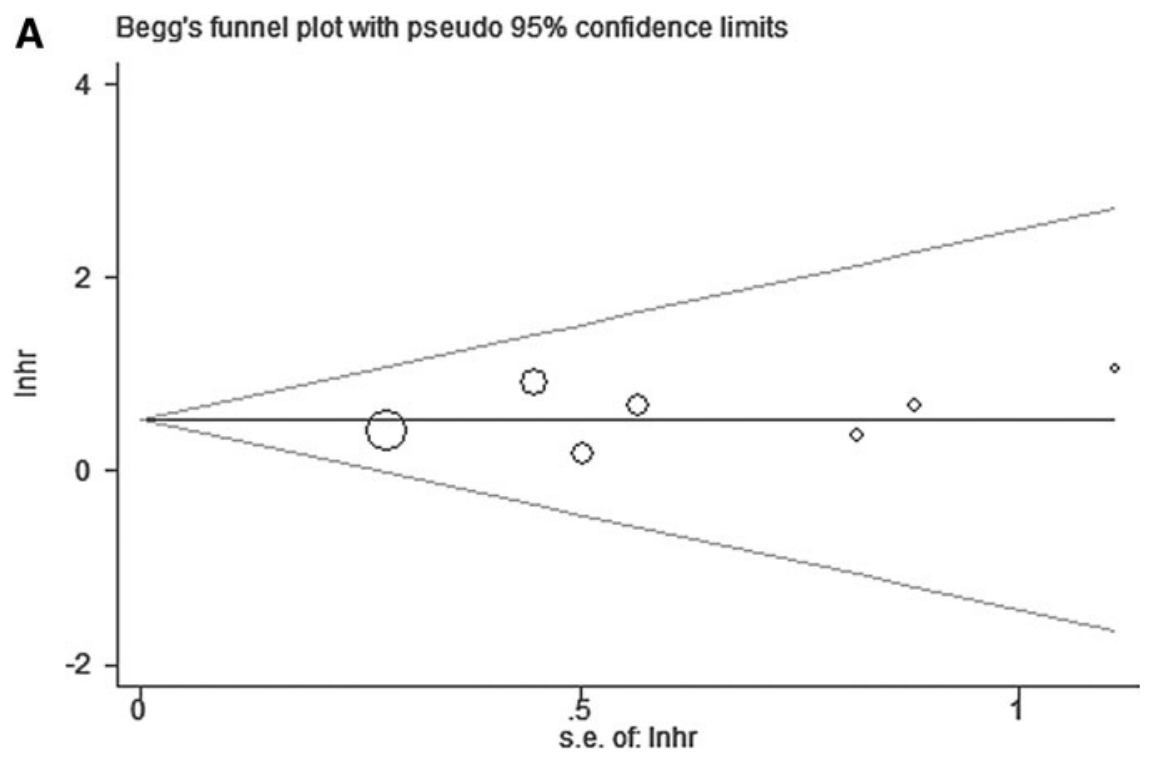

B Begg's funnel plot with pseudo $95 \%$ confidence limits

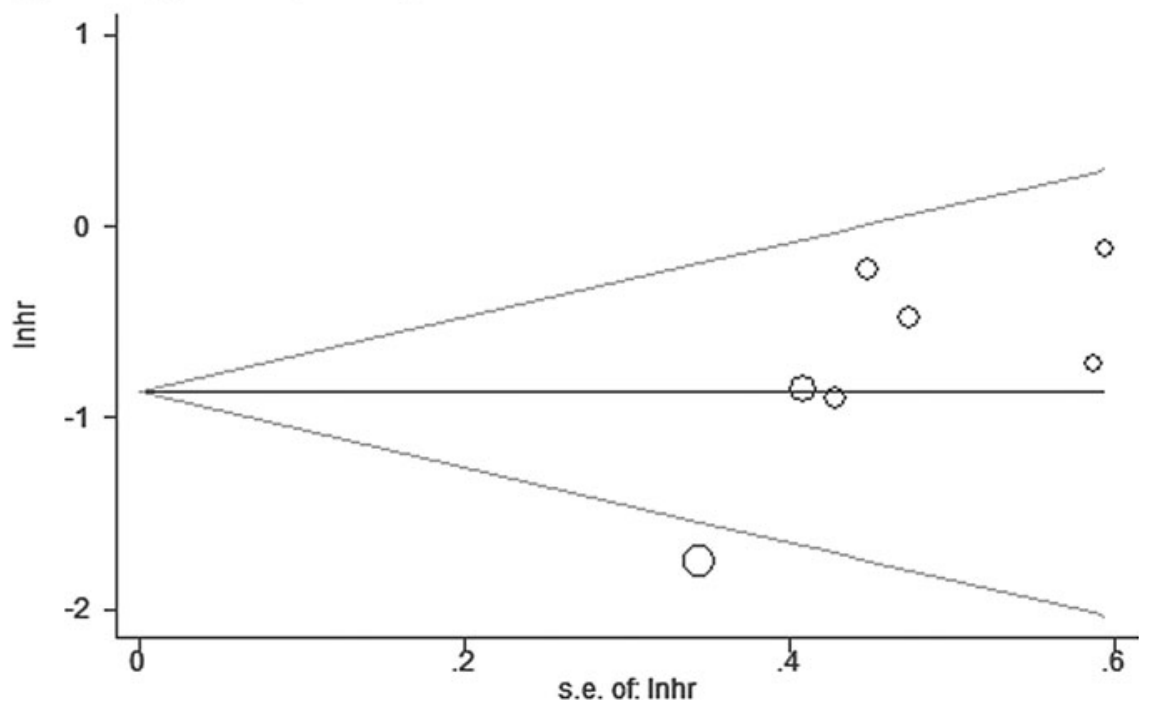

et al. (2017) identified that 280 circRNAs were differentially expressed (Chen et al., 2017). In BC, there are tens of thousands of circRNAs, among which thousands have differential expressions (Li et al., 2017).

Zhong et al. identified the abnormal expression of 469 circRNAs in $\mathrm{BC}$ by comparing their expression with normal tissues using microarray chips. Among these circRNAs, 285 were upregulated and 184 were downregulated. They confirmed six of these circRNAs as being significantly differently expressed using quantitative real-time polymerase chain reaction (qRT-PCR). Subsequently, in vivo and in vitro experiments confirmed that overexpression of one of these, circTCF25, could downregulate miR-103a-3p and miR-107, thereby increasing the expression of cyclin dependent kinase 6 (CDK6) and promoting BC cell proliferation and migration (Wang et al., 2017). In this meta-analysis, 14 studies were included in a total of $1,430 \mathrm{BC}$ cases, revealing the relationship between the expression of different types of circRNA and the clinicopathological and prognostic significance of BC. As different circRNAs play different roles in BC, 7 of the 14 studies we included associated with better clinicopathological features and the remaining 7 are opposite. We divided them into two groups for analysis. We found that $\mathrm{BC}$ patients with high expression of circRNA (circMYLK, circASXL1, hsa_circ_0000144, CircVANGL1, circGprc5a, circZFR, and circ-cTFRC) associated with poor clinicopathological features showed inferior OS (OS: HR $=1.69 ; 95 \%$ CI: 1.17-2.44). circRNA (circMTO1, circ-ITCH, circUBXN7, circFNDC3B, circ-ZKSCAN1, circPICALM, and circACVR2A) with a high expression that associated with better clinicopathological features has a more favorable OS (OS: $\mathrm{HR}=0.42$; 95\% CI: 0.30-0.58). These results suggest that certain circRNAs in BC patients may have a significant prognostic value in OS. Our results further show that some circRNAs (circMYLK, circASXL1, hsa_circ_0000144, Circ-VANGL1, circGprc5a, circZFR, and circ-cTFRC) associated with poorer TNM staging, histological grading, lymph node metastasis, and recurrence in $\mathrm{BC}$, while other circRNAs (circMTO1, circ-ITCH, circUBXN7, circFNDC3B, circ-ZKSCAN1, circPICALM, and circACVR2A) 
are related to clinical tumor characteristics such as better TNM staging, histological grade, and lymph node metastasis. However, we found that no matter which type of circRNAs are highly expressed, they are not related to the clinicopathological characteristics of age, sex, tumor size, and vascular invasion. All these results suggest that the abnormally expressed circRNAs may be related to the clinical prognosis of $\mathrm{BC}$ patients.

In our research, circZFR was identified as one of the highly expressed circRNAs related to tumor recurrence and poor prognoses in multiple cancers. In nonsmall-cell lung carcinoma (NSCLC), Zhang et al. (2019b) showed that expression was significantly upregulated in cancer tissues and cell lines. The knock down of circZFR significantly inhibited cell proliferation, migration, and invasion of NSCLC cells in vitro. Moreover, it was demonstrated that circZFR acted as a sponge to absorb miRNA-101-3p and to promote cullin 4B expression. Similarly, Tan et al. (2019) found that circZFR is highly expressed in hepatocellular carcinoma (HCC) tissues, and their knockdown of circZFR significantly inhibited the proliferation and epithelial/mesenchymal transition of HCC cells. Kaplan/Meier analyses and log-rank tests showed that elevated expression of circZFR was related to poor prognoses of HCC patients. In terms of mechanism, they found that circZFR enhanced the expression of catenin beta 1 (CTNNB1) by sponging miR-3619-5p, thereby activating the Wnt/ $\beta$-catenin pathway, which is known to promote the carcinogenic effect in HCC. In BC, Zhang et al. (2019c) found that the expression of circZFR was increased, and that its expression levels were positively correlated with patients' TNM stage, lymphatic metastases, recurrence, and poor OS. They showed that circZFR could promote BC cell growth, migration, and invasion by binding to miR-377 and acting as a sponge to promote zinc finger E-box binding homeobox 2 (ZEB2) expression. These results indicate that circZFR may be a biomarker of $\mathrm{BC}$ and that ZEB2 may become a target for the treatment of $\mathrm{BC}$.

Although we made every effort to ensure the authenticity and reliability of our research, this meta-analysis is not without its limitations. First, due to the limited amount of literature collected, the number of cases that were ultimately included was relatively small. Second, when the original literature that was included did not directly indicate the HR value, we calculated HRs from the Kaplan/Meier curve (Parmar et al., 1998), which may also introduce potential biases. Therefore, it is important that additional primary research on these circRNAs be conducted through larger BC patient cohort studies.

\section{Conclusions}

In conclusion, our research shows that abnormally expressed circRNAs are related to the clinicopathological features of $\mathrm{BC}$ and could serve as prognostic biomarkers for $\mathrm{BC}$ patients.

\section{Author Disclosure Statement}

No competing financial interests exist.

\section{Funding Information}

No funding was received for this article.

\section{References}

Antoni S, Ferlay J, Soerjomataram I, et al. (2017) Bladder cancer incidence and mortality: a global overview and recent trends. Eur Urol 71:96-108.

Bi J, Liu H, Dong W, et al. (2019) Circular RNA circZKSCAN1 inhibits bladder cancer progression through miR-1178-3p/p21 axis and acts as a prognostic factor of recurrence. Mol Cancer 18:133-133.

Burger M, Catto JWF, Dalbagni G, et al. (2013) Epidemiology and risk factors of urothelial bladder cancer. Eur Urol 63:234-241.

Chen L, Zhang S, Wu J, et al. (2017) circRNA_100290 plays a role in oral cancer by functioning as a sponge of the miR-29 family. Oncogene 36:4551-4561.

Cong L, Yang Q, Hu C, et al. (2019) Current status of functional studies on circular RNAs in bladder cancer and their potential role as diagnostic and prognostic biomarkers: a review. Med Sci Monit 25:3425-3434.

Di Meo A, Bartlett J, Cheng Y, et al. (2017) Liquid biopsy: a step forward towards precision medicine in urologic malignancies. Mol Cancer 16:80-80.

Dong W, Bi JM, Liu HW, et al. (2019) Circular RNA ACVR2A suppresses bladder cancer cells proliferation and metastasis through miR-626/EYA4 axis. Mol Cancer 18:95-95.

Egger M, Davey Smith G, Schneider M, et al. (1997) Bias in meta-analysis detected by a simple, graphical test. BMJ 315 : 629-634.

Gebert LFR, MacRae IJ (2019) Regulation of microRNA function in animals. Nat Rev Mol Cell Biol 20:21-37.

Gu C, Zhou N, Wang Z, et al. (2018) circGprc5a promoted bladder oncogenesis and metastasis through Gprc5a-targeting peptide. Mol Ther Nucleic Acids 13:633-641.

Huang W, Lu Y, Wang F, et al. (2018) Downregulation of circular RNA hsa_circ_0000144 inhibits bladder cancer progression via stimulating miR-217 and suppressing RUNX2 expression. Gene 678:337-342.

Li Y, Zheng F, Xiao X, et al. (2017) CircHIPK3 sponges miR558 to suppress heparanase expression in bladder cancer cells. EMBO Rep 18:1646-1659.

Li YX, Wan B, Liu L, et al. (2019a) Circular RNA circMTO1 suppresses bladder cancer metastasis by sponging miR-221 and inhibiting epithelial-to-mesenchymal transition. Biochem Biophys Res Commun 508:991-996.

Li Z, Ruan Y, Zhang H, et al. (2019b) Tumor-suppressive circular RNAs: mechanisms underlying their suppression of tumor occurrence and use as therapeutic targets. Cancer Sci 110:3630-3638.

Liu H, Chen D, Bi J, et al. (2018a) Circular RNA circUBXN7 represses cell growth and invasion by sponging miR-1247-3p to enhance B4GALT3 expression in bladder cancer. Aging (Albany NY) 10:2606-2623.

Liu HW, Bi JM, Dong W, et al. (2018b) Invasion-related circular RNA circFNDC3B inhibits bladder cancer progression through the miR-1178-3p/G3BP2/SRC/FAK axis. Mol Cancer 17:161-161.

Martinez Rodriguez RH, Buisan Rueda O, Ibarz L (2017) Bladder cancer: present and future. Med Clin (Barc) 149:449-455.

Meng S, Zhou H, Feng Z, et al. (2017) CircRNA: functions and properties of a novel potential biomarker for cancer. Mol Cancer 16:94-94.

Parmar MK, Torri V, Stewart L (1998) Extracting summary statistics to perform meta-analyses of the published literature for survival endpoints. Stat Med 17:2815-2834.

Patop IL, Wüst S, Kadener S (2019) Past, present, and future of circRNAs. EMBO J 38:e100836. 
Salmena L, Poliseno L, Tay Y, et al. (2011) A ceRNA hypothesis: the Rosetta Stone of a hidden RNA language? Cell 146:353-358.

Salzman J (2016) Circular RNA expression: its potential regulation and function. Trends Genet 32:309-316.

Sanger HL, Klotz G, Riesner D, et al. (1976) Viroids are singlestranded covalently closed circular RNA molecules existing as highly base-paired rod-like structures. Proc Natl Acad Sci U S A 73:3852-3856.

Siegel RL, Miller KD, Jemal A (2017) Cancer statistics, 2017. CA Cancer J Clin 67:7-30.

Slack FJ, Chinnaiyan AM (2019) The role of non-coding RNAs in oncology. Cell 179:1033-1055.

Stang A (2010). Critical evaluation of the Newcastle-Ottawa scale for the assessment of the quality of nonrandomized studies in meta-analyses. Eur J Epidemiol 25:603-605.

Su HW, Tao T, Yang Z, et al. (2019) Circular RNA cTFRC acts as the sponge of MicroRNA-107 to promote bladder carcinoma progression. Mol Cancer 18:27-27.

Tan A, Li Q, Chen L (2019) CircZFR promotes hepatocellular carcinoma progression through regulating miR-3619$5 \mathrm{p} / \mathrm{CTNNB} 1$ axis and activating $\mathrm{Wnt} / \beta$-catenin pathway. Arch Biochem Biophys 661:196-202.

Tang G, Xie WQ, Qin C, et al. (2017) Expression of circular RNA circASXL1 correlates with TNM classification and predicts overall survival in bladder cancer. Int J Clin Exp Pathol 10:8495-8502.

Wang D, Yang S, Wang H, et al. (2018a) The progress of circular RNAs in various tumors. Am J Transl Res 10:1571-1582.

Wang K, Sun Y, Tao W, et al. (2017) Androgen receptor (AR) promotes clear cell renal cell carcinoma (ccRCC) migration and invasion via altering the circHIAT1/miR-195-5p/29a3p/29c-3p/CDC42 signals. Cancer Lett 394:1-12.

Wang M, Yang Y, Xu J, et al. (2018b) CircRNAs as biomarkers of cancer: a meta-analysis. BMC Cancer 18:303-303.

Yan D, Dong W, He Q, et al. (2019) Circular RNA circPICALM sponges miR-1265 to inhibit bladder cancer metastasis and influence FAK phosphorylation. EBioMedicine 48:316-331.

Yang CD, Yuan WB, Yang X, et al. (2018) Circular RNA circITCH inhibits bladder cancer progression by sponging miR-
17/miR-224 and regulating p21, PTEN expression. Mol Cancer 17:19-19.

Yao RW, Wang Y, Chen LL (2019) Cellular functions of long noncoding RNAs. Nat Cell Biol 21:542-551.

Zeng ZH, Zhou WM, Duan LX, et al. (2019) Circular RNA circ-VANGL1 as a competing endogenous RNA contributes to bladder cancer progression by regulating miR-6053p/VANGL1 pathway. J Cell Physiol 234:3887-3896.

Zhang B, Chen M, Jiang N, et al. (2019a) A regulatory circuit of circ-MTO1/miR-17/QKI-5 inhibits the proliferation of lung adenocarcinoma. Cancer Biol Ther 20:1127-1135.

Zhang H, Wang X, Hu B, et al. (2019b) Circular RNA ZFR accelerates non-small cell lung cancer progression by acting as a miR-101-3p sponge to enhance CUL4B expression. Artif Cells Nanomed Biotechnol 47:3410-3416.

Zhang WY, Liu QH, Wang TJ, et al. (2019c) CircZFR serves as a prognostic marker to promote bladder cancer progression by regulating miR-377/ZEB2 signaling. Biosci Rep 39: BSR20192779.

Zheng J, Liu X, Xue Y, et al. (2017) TTBK2 circular RNA promotes glioma malignancy by regulating miR-217/HNF1 $\beta /$ Derlin-1 pathway. J Hematol Oncol 10:52.

Zhong Z, Huang M, Lv M, et al. (2017) Circular RNA MYLK as a competing endogenous RNA promotes bladder cancer progression through modulating VEGFA/VEGFR2 signaling pathway. Cancer Lett 403:305-317.

Address correspondence to: Ting Sun, MD

Department of Urology

The First Affiliated Hospital of Nanchang University No. 17, Yongwai Center Street

Donghu District Nanchang 330006 Jiangxi China

E-mail: journal_123@163.com 